\title{
Myofibroblasts Alter Tension and Strain of Cardiac Fiber:
}

\section{A Computational Study}

\author{
Heqing Zhan ${ }^{1}$, Jingtao Zhang ${ }^{2}$ \\ ${ }^{1}$ College of Medical Information, Hainan Medical University, Haikou, 571199, China \\ ${ }^{2}$ Cardiac Arrhythmia Center, Fuwai Hospital, National Center for Cardiovascular Diseases, \\ Beijing, 100037, China
}

\begin{abstract}
In heart pathological conditions, fibroblasts proliferate and differentiate into myofibroblasts (Mfbs). This study aimed to investigate the role of Mfbs on the mechanical contraction of cardiac fiber. Mathematical modeling was done using a combination of (1) the Maleckar et al. model of the human atrial myocyte, (2) the MacCannell et al. active model of the human cardiac $M f b$, (3) our formulation of $I_{N a \_m y o f b}$ based upon experimental findings from Chatelier et al., and (4) the Hill three-element rheological scheme of a single segment of cardiac fiber. For Mfb-myocyte coupling, different ratios of myocytes to Mfbs and gap-junctional conductances were set based on available physiological data. Both isometric contraction and isotonic contraction were considered to illustrate the effect of Mfbs on cardiac fiber's tension and strain. The results showed that (1) $M f b s$ decreased $A P D_{50}$ and increased $V_{\text {rest }}$ depolarization, (2) Mfbs regulated myocyte peak force and (3) Mfbs reduced the fiber peak force in isometric contraction and the fiber peak strain in isotonic contraction. The identified effects demonstrated that Mfbs play an important role of modulating cardiac mechanical behavior. It should be considered in future pathological cardiac mathematical modeling, such as atrial fibrillation and cardiac fibrosis.
\end{abstract}

\section{Introduction}

As one type of connective tissues, cardiac fibroblasts account for approximately $75 \%$ of all cardiac cells but only contribute $5-10 \%$ of the total mammalian heart volume due to their small size [1]. During pathophysiological challenges, such as cardiac fibrosis, myofibroblast (Mfb) cell population emerges as the subpopulation of fibroblasts that are responsible for tissue remodeling [2]. Fibrosis, characterized by fibroblast proliferation and collagen production, is strongly correlated with atrial and ventricular tachyarrhythmias and sudden cardiac death [3].

Recently, clinical data and simulation studies have verified that Mfbs have several currents like cardiac myocytes. One of them is the current through voltagegated sodium channels in Mfbs ( $\left.I_{\mathrm{Na} \_ \text {myofb }}\right)$ [4]. Computational models of atrial fibrosis have been used to investigate how fibroblasts modulate cardiac myocyte electrophysiology and mechanics. They showed that coupling of Mfbs to atrial myocytes resulted in shorter duration of the action potential (APD), slower conduction, and spiral wave breakups [5,6]. Meanwhile, they found that fibroblast alignment parallel to a strain cue provided negative feedback to radical changes in local fiber orientations [7, 8]. These studies have discussed cardiac myocyte function in a lot of ways, yet they have not considered $I_{\text {Na_myofb }}$ in Mfb-myocyte $(\mathrm{Mfb}-\mathrm{M})$ coupling, which could influence $\mathrm{Mfb}$ properties and contribute to electromechanical coupling in cardiac pathologies.

In previous study, we have found that the Nav $1.5 \alpha$ subunit, which generates $I_{\mathrm{Na} \_ \text {myofb}}$, regenerated action potentials (APs) in myocytes and Mfbs [9]. In this study, we aimed to investigate the role of $\mathrm{Mfbs}$ on the mechanical contraction of cardiac fiber. $I_{\text {Na_myofb }}$ was considered in the process. Simulation results of 1D human atrial fiber mechanical dynamics with different number of coupled Mfbs and gap-junctional conductances $\left(G_{\text {gap }}\right)$ were compared.

\section{Meterials and Methods}

Mathematical modeling was done using a combination of (1) the Maleckar et al. model of the human atrial myocyte, (2) the MacCannell et al. active model of the human cardiac myofibroblast, (3) our formulation of $I_{\text {Na_myofb }}$ based upon experimental findings from Chatelier et al., and (4) the Hill three-element rheological scheme of a single segment of cardiac fiber. An overview of the simulations was given as follows.

\subsection{Mfb-M coupling}

According to [10], the differential equations for the membrane potential of cardiac $\mathrm{Mfb}$ and myocyte are given by 


$$
\begin{aligned}
& \frac{d V_{\mathrm{Mfb}, \mathrm{i}}}{d t}=-\frac{1}{\mathrm{C}_{\mathrm{m}, \mathrm{Mfb}}}\left(I_{\mathrm{Mfb}, \mathrm{i}}\left(V_{\mathrm{Mfb}, \mathrm{i}}, t\right)\right. \\
& \left.\quad+G_{\mathrm{gap}}\left(V_{\mathrm{Mfb}, \mathrm{i}}-V_{\mathrm{M}}\right)\right) \\
& \frac{d V_{\mathrm{M}}}{d t}=-\frac{1}{\mathrm{C}_{\mathrm{m}, \mathrm{M}}}\left(I_{\mathrm{M}}\left(V_{\mathrm{M}}, t\right)+\sum_{\mathrm{i}=1}^{\mathrm{n}} G_{\mathrm{gap}}\left(V_{\mathrm{M}}-V_{\mathrm{Mfb}, \mathrm{i}}\right)\right)
\end{aligned}
$$

where $V_{\mathrm{Mfb}, \mathrm{i}}$ and $V_{\mathrm{M}}$ represent the transmenbrane potential of the ith coupled Mfb and the human atrial myocyte, respectively; $C_{m, M f b}$ and $C_{m, M}$ represent the membrane capacitance of the Mfb and the myocyte, respectively; $I_{\mathrm{Mfb}, \mathrm{i}}$ and $I_{\mathrm{M}}$ represent the transmenbrane current of the $i$ th coupled Mfb and the human atrial myocyte, respectively; $G_{\text {gap }}$ represents the gap-junctional conductance; and $\mathrm{n}$ is the total number of coupled Mfbs.

\subsection{Model of the human atrial myocyte}

The mathematical model from Maleckar et al. is implemented in this study [11], which is based on experimental data and has correctly replicated APD restitution of the adult human atrial myocyte.

The model 4 of isometric force generation in cardiac myofilaments proposed by Rice et al. is applied to model the $\mathrm{Ca}^{2+}$-force relation in the present study [12].

\subsection{Model of human atrial Mfbs}

The active electrophysiological model of atrial $\mathrm{Mfb}$ described by Maleckar et al is used in the present study [11]. In addition, $I_{\text {Na_Mfb }}$ is added in the Mfb model. According to our previous work [9], equations of $I_{\mathrm{Na} \_\mathrm{Mfb}}$ is given by

$$
\begin{gathered}
I_{\mathrm{Na} \_\mathrm{Mfb}}=\bar{g}_{\mathrm{Na}, \mathrm{Mfb}} m_{\mathrm{Mfb}} j_{\mathrm{Mfb}}^{0.12}\left(V_{\mathrm{Mfb}}-E_{\mathrm{Na}, \mathrm{Mfb}}\right) \\
E_{\mathrm{Na}, \mathrm{Mfb}}=\frac{\mathrm{RT}}{\mathrm{F}} \log \frac{\left[\mathrm{Na}^{+}\right]_{\mathrm{c}, \mathrm{Mfb}}}{\left[\mathrm{Na}^{+}\right]_{\mathrm{i}, \mathrm{Mfb}}}
\end{gathered}
$$

where $\bar{g}_{\mathrm{Na}, \mathrm{Mfb}}$ is the maximum conductance of $I_{\mathrm{Na} \_\mathrm{Mfb}}$; $E_{\mathrm{Na} \_\mathrm{Mfb}}$ is the Nernst potential for $\mathrm{Na}^{+}$ions; $\left[\mathrm{Na}^{+}\right]_{\mathrm{c}, \mathrm{Mfb}}$ and $\left[\mathrm{Na}^{-}\right]_{i, \mathrm{Mfb}}$ are the Mfb extracellular and intracellular $\mathrm{Na}^{+}$ concentration, respectively; $m_{\mathrm{Mfb}}$ and $j_{\mathrm{Mfb}}$ are the activation and inactivation parameters, respectively.

\subsection{Mechanical behavior of a cardiac fiber with inserted Mfbs}

The mechanical behavior of a single segment in our model is based on the classical three-element rheological scheme [13]. It consists of a contractile element (CE), a series elastic element (SE) and a parallel elastic element (PE). Cardiac fiber is modeled as a string of segments coupled in series. Each segment is regarded as a myocyte/Mfbs complex. Mfbs are coupled to each myocyte by longitudinal connection, which distributed along the long axis of segments. The electrical component is governed by the parabolic partial differential monodomain equation. The mechanical component is described as follows [14],

$$
\begin{gathered}
F_{\text {segment }}^{n}=F_{\text {fiber }} \\
\lambda_{\text {fiber }}=\frac{L}{L_{0}}=\frac{\sum_{n \epsilon N} l_{\mathrm{PE}}^{n}}{\sum_{n \epsilon N} l_{\mathrm{PE}_{0}}^{n}}
\end{gathered}
$$

where $F_{\text {segment }}$ is the total force generated by the segment. $F_{\text {fiber }}$ is the force generated by the fiber, $\lambda_{\text {fiber }}$ is the stretch ratio of the fiber, $L$ denotes the actual fiber length, $L_{0}$ is the reference length, $l_{\mathrm{PE}}^{n}$ and $l_{\mathrm{PE}_{0}}^{n}$ represent the reference length and the actual length of the PE of segment $n$.

\subsection{Simulation protocol and numerical methods}

We performed the impulse propagation along a $5-\mathrm{cm}$ long homogeneous fiber with isometric contraction and isotonic contraction to illustrate the effects of Mfbs on cardiac fiber mechanical properties. Mfb-M ratio was 1,2 and 3, respectively. $G_{\text {gap }}$ was $0.5,3$ and $8 \mathrm{nS}$, respectively.

To ensure the coupled system reached steady-state, stimulation was repeated for 20 cycles. Results from the last cycle in each simulation were used. All state variables of the coupled model were updated by means of the forward Euler method. The time step was set to be 10 $\mu$ s to ensure numerical accuracy and stability.

\section{Results}

\subsection{Effects of Mfb on myocyte $\mathrm{AP}$ and force}
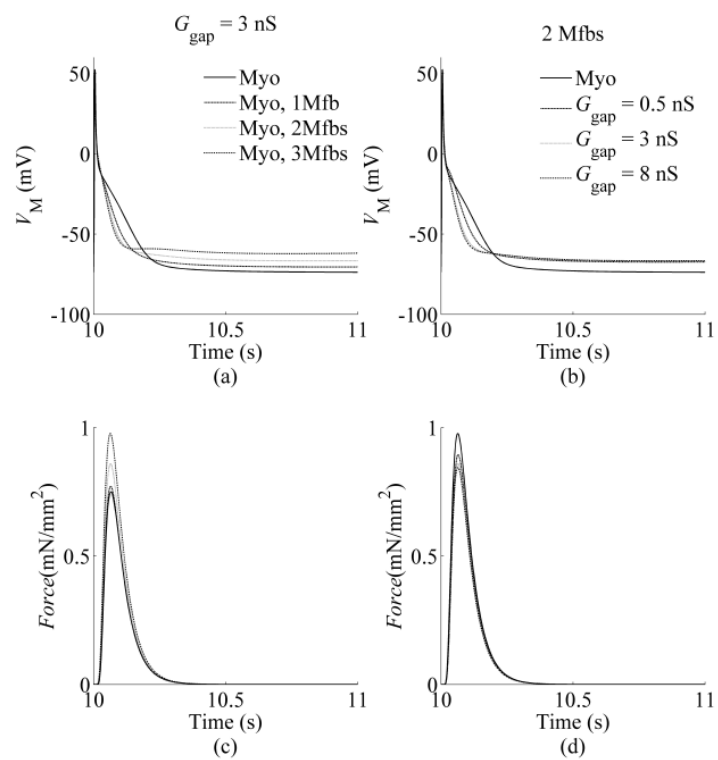

Figure 1. Effects of Mfb on myocyte AP and force. In (a) and (c), pure myocyte (solid), myocyte with $1 \mathrm{Mfb}$ (dashed), myocyte with $2 \mathrm{Mfbs}$ (dotted), myocyte with $3 \mathrm{Mfbs}$ (dashdot), $G_{\text {gap }}=3 \mathrm{nS}$. In (b) and (d), pure myocyte (solid), $G_{\text {gap }}=0.5 \mathrm{nS}$ 
(dashed), $G_{\text {gap }}=3 \mathrm{nS}$ (dotted), $G_{\text {gap }}=8 \mathrm{nS}$ (dashdot), and myocyte was coupled with 2 Mfbs.

Figure 1 showed AP and force in myocytes with different number of coupled Mfbs and $G_{\text {gap }}$. On electrophysiology, increased Mfbs and $G_{\text {gap }}$ resulted in gradually decreased $\mathrm{APD}$ at $50 \%$ repolarization $\left(\mathrm{APD}_{50}\right)$, and increased the resting myocyte membrane potential $\left(V_{\text {rest }}\right)$ depolarization (Figure 1a and 1b). Comparing to the control (no Mfbs), $\mathrm{APD}_{50}$ was decreased by $32 \%$ (1 $\mathrm{Mfb}), 48 \%$ ( $2 \mathrm{Mfbs}$ ) and $50 \%$ (3 Mfbs), and by $25 \%$ ( 0.5 $\mathrm{nS}), 48 \%(3 \mathrm{nS})$ and $49 \%(8 \mathrm{nS})$, respectively. $V_{\text {rest }}$ was increased by $13.8 \%$ ( $1 \mathrm{Mfb}), 29.3 \%$ (2 Mfbs) and $44.8 \%$ (3 Mfbs), and by $20.1 \%(0.5 \mathrm{nS}), 24.3 \%$ (3 nS) and $28 \%$ $(8 \mathrm{nS})$, respectively. On mechanics, peak force increased with increased Mfbs, while decreased with increased $G_{\text {gap }}$ (Figure 1c and 1d). It was increased by $2.7 \%$ (1 Mfb), $14.3 \%$ (2 Mfbs) and 30.5\% (3 Mfbs), and decreased by $6.7 \%(0.5 \mathrm{nS}), 11.4 \%(3 \mathrm{nS})$ and $13 \%(8 \mathrm{nS})$, respectively.

\subsection{Effects of Mfb on tension and strain of cardiac fiber}
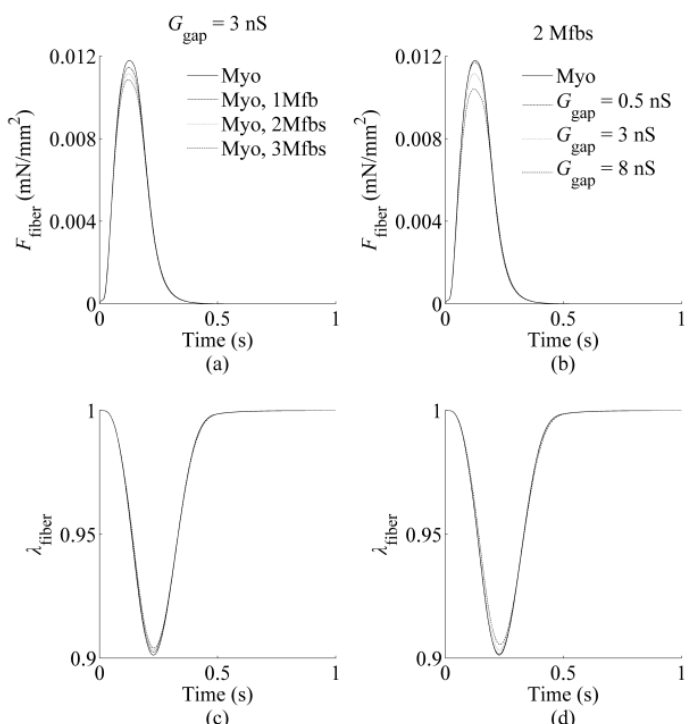

Figure 2. Effects of $\mathrm{Mfb}$ on $F_{\text {fiber }}$ (isometric contraction) and $\lambda_{\text {fiber }}$ (isotonic contraction) of cardiac fiber. In (a) and (c), pure myocyte (solid), myocyte with $1 \mathrm{Mfb}$ (dashed), myocyte with 2 Mfbs (dotted), myocyte with $3 \mathrm{Mfbs}$ (dashdot), $G_{\text {gap }}=3$ nS. In (b) and (d), pure myocyte (solid), $G_{\text {gap }}=0.5 \mathrm{nS}$ (dashed), $G_{\text {gap }}=3 \mathrm{nS}$ (dotted), $G_{\text {gap }}=8 \mathrm{nS}$ (dashdot), and myocyte was coupled with $2 \mathrm{Mfbs}$.

In Figure 2, traces of cardiac fiber tension and stretch ratio were presented for simulations of isometric contraction with sarcomere length of $1.78 \mu \mathrm{m}$ and isotonic contraction with applied force of $10 \mathrm{mN} / \mathrm{mm}^{2}$.

In isometric contraction (Figure $2 \mathrm{a}$ and $2 \mathrm{~b}$ ), peak $F_{\text {fiber }}$ both decreased when the number of $\mathrm{Mfbs}$ and $G_{\text {gap }}$ increased. Comparing to the control (no Mfbs), it was decreased by $2.9 \%$ ( $1 \mathrm{Mfb}), 5.5 \%$ (2 Mfbs) and $7.9 \%$ (3 Mfbs), and by $0.8 \%(0.5 \mathrm{nS}), 5.5 \%(3 \mathrm{nS})$ and $11.8 \%(8$ $\mathrm{nS})$, respectively.

In isotonic contraction (Figure $2 \mathrm{c}$ and $2 \mathrm{~d}$ ), the minimum of $\lambda_{\text {fiber }}$ also both decreased when the number of Mfbs and $G_{\text {gap }}$ increased. It was decreased by $1.2 \%$ (1 $\mathrm{Mfb}), 2.1 \%$ (2 Mfbs) and 2.9\% (3 Mfbs), and by $0.5 \%$ $(0.5 \mathrm{nS}), 2.1 \%(3 \mathrm{nS})$ and $4.3 \%(8 \mathrm{nS})$, respectively.

\section{Discussion}

This study investigated the roles of Mfb in myocyte $\mathrm{AP}$ and in 1D cardiac fiber mechanical behavior. Numerical simulations of the coupled Mfb-M system were performed by employing a combination of models of the human atrial myocyte and $\mathrm{Mfb}$ (including $I_{\mathrm{Na} \_\mathrm{Mfb}}$ ), models of $\mathrm{Ca}^{2+}$-force relation and myocyte mechanical segment. Specifically, effects of Mfbs with different number of coupled $\mathrm{Mfbs}$ and $G_{\text {gap }}$ on atrial fiber properties were investigated. Coupling Mfbs could result in: (1) decreased $\mathrm{APD}_{50}$ and increased $V_{\text {rest }}$ depolarization, (2) increased the peak force with increased Mfbs, and decreased the peak force with increased $G_{\text {gap }}$, and (3) decreased the peak value of $F_{\text {fiber }}$ in isometric contraction and the minimum of $\lambda_{\text {fiber }}$ in isotonic contraction.

Our simulations showed a depolarizing effect of coupled Mfbs on $V_{\text {rest }}$ of atrial myocytes. Figure 1 showed a maximum depolarization of $14 \mathrm{mV}$ for a myocyte with 3 Mfbs. Previous experimental studies have shown the depolarization of neonatal rat ventricular cardiomyocyte strands when Mfbs interacted with myocytes. Modeling studies have also shown that fibroblasts depolarized coupled myocytes. MacCannell et al verified that $V_{\text {rest }}$ of the coupled myocyte was depolarized slightly $(\sim 2.7 \mathrm{mV})$ for up to 10 fibroblasts per myocyte, and was insensitive to $G_{\text {gap }}[10]$. However, Maleckar et al reported that a $G_{\text {gap }}$ of $8 \mathrm{nS}$ and two active 1 fibroblast resulted in a $V_{\text {rest }}$ elevation of $8.3 \mathrm{mV} \mathrm{[15].}$

Coupling human atrial myocytes with Mfbs also resulted in diverse effect on AP morphology during repolarization. It has been found that fibroblasts functioned as strong current sources at rest and as both sources and sinks during the AP when they employed a myocyte-fibroblast coupling model with a high $G_{\text {gap }}$ [15]. In that study, the prolongation of repolarization was emerged early in the AP, and plateau was prolonged or shortened depending on both the fibroblast $V_{\text {rest }}$ and number of coupled fibroblasts. Ventricular myocyte AP was compared when fibroblasts with a membrane capacitance of $6 \mathrm{pF}$ or $60 \mathrm{pF}$ were coupled to a myocyte. It showed that APD was shortened much more when large fibroblasts were coupled. In our simulations, increasing the number of Mfbs and $G_{\text {gap }}$ decreased $\mathrm{APD}_{50}$.

For mechanics, previous studies have verified that 
mechanical cues activated cardiac Mfbs [16]. Mfbs were regarded as a critical determinant of cardiac mechanics. Computational modeling was used to demonstrate the acute mechanical effects on cardiac fibroblast structure and organization [17]. It was verified that an axial strain environment could guide fibroblast proliferation, orientation and migration [18]. Several groups have demonstrated that cellular organization was tightly linked to the mechanical feedback loop between cells and matrix [7]. This studies were about the stretch-induced responses of quiescent cardiac Mfbs. However, the inverse process, the Mfbs-induced responses of cardiac mechanics, has not yet been widely understood. Our results showed that coupling Mfbs changed cardiac fiber mechanical properties. Coupling Mfbs both decreased tension and strain of cardiac fiber.

\section{Summary}

This study demonstrated the effects of Mfbs on cardiac fiber mechanical properties. Our results showed that Mfbs regulated the peak and valley values of fiber mechanical parameters in both isometric contraction and isotonic contraction. The effects proved that Mfbs should be considered in future pathological cardiac mechanical mathematical modeling, such as atrial fibrillation and cardiac fibrosis.

\section{Acknowledgements}

This project is supported by the National Natural Science Foundation of China (81501557).

\section{References}

[1] Banerjee I, J W Fuseler, R L Price, T K Borg, and T A Baudino. Determination of cell types and numbers during cardiac development in the neonatal and adult rat and mouse. Am J Physiol Heart Circ Physiol, 2007; 293(3): H1883-91.

[2] Ivey M J and M D Tallquist. Defining the Cardiac Fibroblast. Circ J, 2016; 80(11): 2269-2276.

[3] Pakshir P and B Hinz. The big five in fibrosis: Macrophages, myofibroblasts, matrix, mechanics, and miscommunication. Matrix Biol, 2018; 68-69: 81-93.

[4] Koivumäki J T, R B Clark, D Belke, C Kondo, P W M Fedak, M M C Maleckar, and W R Giles. $\mathrm{Na}(+)$ current expression in human atrial myofibroblasts: identity and functional roles. Frontiers in physiology, 2014; 5: 275-275.

[5] Ashihara T, R Haraguchi, K Nakazawa, T Namba, T Ikeda, Y Nakazawa, T Ozawa, M Ito, M Horie, and N A Trayanova. The role of fibroblasts in complex fractionated electrograms during persistent/permanent atrial fibrillation: implications for electrogram-based catheter ablation. Circulation Research, 2012; 110(2): 275-84.

[6] McDowell K S, F Vadakkumpadan, R Blake, J Blauer, G Plank, R S Macleod, and N A Trayanova. Mechanistic inquiry into the role of tissue remodeling in fibrotic lesions in human atrial fibrillation. Biophys J, 2013; 104(12): 2764-73.

[7] Checa S, M K Rausch, A Petersen, E Kuhl, and G N Duda. The emergence of extracellular matrix mechanics and cell traction forces as important regulators of cellular selforganization. Biomechanics and modeling in mechanobiology, 2015; 14(1): 1-13.

[8] Rouillard A D and J W Holmes. Coupled agent-based and finite-element models for predicting scar structure following myocardial infarction. Prog Biophys Mol Biol, 2014; 115(2-3): 235-43.

[9] Zhan H, J Zhang, J Lin, and G Han. Effects of $\mathrm{Na}(+)$ Current and Mechanogated Channels in Myofibroblasts on Myocyte Excitability and Repolarization. Computational and mathematical methods in medicine, 2016; 2016: 6189374-6189374.

[10] MacCannell K A, H Bazzazi, L Chilton, Y Shibukawa, R B Clark, and $\mathrm{W} R$ Giles. A mathematical model of electrotonic interactions between ventricular myocytes and fibroblasts. Biophysical Journal, 2007; 92(11): 41214132.

[11] Maleckar M M, J L Greenstein, W R Giles, and N A Trayanova. $\mathrm{K}+$ current changes account for the rate dependence of the action potential in the human atrial myocyte. Am J Physiol Heart Circ Physiol, 2009; 297(4): H1398-410.

[12] Rice J J, M S Jafri, and R L Winslow. Modeling shortterm interval-force relations in cardiac muscle. Am $\mathrm{J}$ Physiol Heart Circ Physiol, 2000; 278(3): H913-31.

[13] Solovyova O, L Katsnelson, S Guriev, L Nikitina, Y Protsenko, S Routkevitch, and V Markhasin. Mechanical inhomogeneity of myocardium studied in parallel and serial cardiac muscle duplexes: experiments and models. Chaos Solitons \& Fractals, 2002; 13(8): 1685-1711.

[14] Kuijpers N H, H M ten Eikelder, P H Bovendeerd, S Verheule, T Arts, and P A Hilbers. Mechanoelectric feedback leads to conduction slowing and block in acutely dilated atria: a modeling study of cardiac electromechanics. Am J Physiol Heart Circ Physiol, 2007; 292(6): H2832-53.

[15] Maleckar M M, J L Greenstein, W R Giles, and N A Trayanova. Electrotonic coupling between human atrial myocytes and fibroblasts alters myocyte excitability and repolarization. Biophys J, 2009; 97(8): 2179-90.

[16] van Putten S, Y Shafieyan, and B Hinz. Mechanical control of cardiac myofibroblasts. J Mol Cell Cardiol, 2016; 93: 133-42.

[17] Wang H, A S Abhilash, C S Chen, R G Wells, and V B Shenoy. Long-range force transmission in fibrous matrices enabled by tension-driven alignment of fibers. Biophys J, 2014; 107(11): 2592-603.

[18] Rouillard A D and J W Holmes. Mechanical regulation of fibroblast migration and collagen remodelling in healing myocardial infarcts. J Physiol, 2012; 590(18): 4585-602.

Address for correspondence.

Heqing Zhan

College of Medical Information, Hainan Medical University, Haikou, 571199, China

E-mail: zhq86zijing@163.com 